Proceedings of the Edinburgh Mathematical Society (2005) 48, 651-671 (C)

DOI:10.1017/S001309150400001X Printed in the United Kingdom

\title{
REPTILES WITH HOLES
}

\author{
FRANCIS JORDAN AND SZE-MAN NGAI \\ Department of Mathematical Sciences, Georgia Southern University, \\ Statesboro, GA 30460-8093, USA \\ (fjordan@georgiasouthern.edu; ngai@gsu.mat.georgiasouthern.edu)
}

(Received 24 February 2004)

\begin{abstract}
Croft, Falconer and Guy asked: what is the smallest integer $n$ such that an $n$-reptile in the plane has a hole? Motivated by this question, we describe a geometric method of constructing reptiles in $\mathbb{R}^{d}$, especially reptiles with holes. In particular, we construct, for each even integer $n \geqslant 4$, an $n$-reptile in $\mathbb{R}^{2}$ with holes. We also answer some questions concerning the topological properties of a reptile whose interior consists of infinitely many components.
\end{abstract}

Keywords: reptile; self-similar set; iterated function system

2000 Mathematics subject classification: Primary 52C20

Secondary $28 \mathrm{~A} 80$

\section{Introduction}

An $n$-reptile (or $n$-rep tile) $T$ in $\mathbb{R}^{d}$ is a compact set with non-empty interior that can be tiled by $n$ congruent tiles, each similar to $T$ (see $[\mathbf{3}, \mathbf{5}]$ ). We assume, as in the literature, that a reptile is the closure of its interior. If the number of pieces $n$ is irrelevant to the discussion, we will simply call an $n$-reptile a reptile.

Reptiles form a special class of self-similar sets. Let $\left\{f_{i}\right\}_{i=1}^{n}$ be an iterated function system (IFS) of contractive similitudes on $\mathbb{R}^{d}$ defined as

$$
f_{i}(x)=\frac{1}{\sqrt[d]{n}} R_{i} x+d_{i}, \quad i=1, \ldots, n,
$$

where $R_{i}$ is an orthogonal transformation, $d_{i} \in \mathbb{R}^{d}$, and the factor $1 / \sqrt[d]{n}$ is the contraction ratio of $f_{i}$. Then there exists a unique compact set $T$ satisfying

$$
T=\bigcup_{i=1}^{n} f_{i}(T)
$$

(see $[\mathbf{4}, \mathbf{7}]) . T$ is called the self-similar set (or $n$-repset, or attractor) defined by $\left\{f_{i}\right\}_{i=1}^{n}$. It follows from (1.1) and the uniqueness of $T$ that $T$ is the closure of its interior. If the interior of $T$ is non-empty, then it follows from (1.1) that $T$ is an $n$-reptile. Note that the 
similarity dimension (see, for example, $[\mathbf{7}])$ of $\left\{f_{i}\right\}_{i=1}^{n}$ is $d$. Thus, the assumption that $T$ has a non-empty interior is equivalent to the requirement that $\left\{f_{i}\right\}_{i=1}^{n}$ satisfies the open set condition [11]. Therefore, $T$ is an $n$-reptile if and only if $T$ is a self-similar set defined by an iterated function system consisting of $n$ similitudes having the same contraction ratio $1 / \sqrt[d]{n}$ and satisfying the open set condition.

Identity (1.1) is equivalent to

$$
\sqrt[d]{n} T=\bigcup_{i=1}^{n}\left(R_{i} T+\sqrt[d]{n} d_{i}\right)
$$

If $T$ is a reptile, then it follows from the above equality and a standard blow-up argument that $\mathbb{R}^{d}$ can be tiled by essentially disjoint congruent copies of $T$ (see, for example, $[\mathbf{8}$, Theorem 1.2]).

We say that a reptile $T \subseteq \mathbb{R}^{d}$ has a hole if the complement of the closure of some component of the interior of $T$ has a bounded component. Answering a question posed by John Conway, Grünbaum gave an example of a 36-reptile in $\mathbb{R}^{2}$ which has a hole (see [3, Figure C17]). In [3, $\S \mathrm{C} 17]$, Croft et al. asked the following question: what is the least $n$ for which an $n$-reptile in the plane has any sort of hole? It has been proved recently by Bandt and Wang [2] and Luo et al. [9] that if the interior of an $n$-reptile in $\mathbb{R}^{2}$ is connected, then the reptile is a topological disc. We will therefore be interested in reptiles whose interiors are disconnected.

In $\S 3$, we construct, for every $m \geqslant 2$, a $2 m$-reptile in $\mathbb{R}^{2}$ that has holes. Define an IFS on $\mathbb{R}^{2}$ by

$$
\begin{aligned}
& f_{i}(x)= \begin{cases}\frac{1}{\sqrt{n}} R\left(\frac{\pi}{2}\right)(x)+\left(i+\frac{1}{2}, 0\right), & -m \leqslant i \leqslant-1, \\
\frac{1}{\sqrt{n}} R\left(-\frac{\pi}{2}\right)(x)+\left(i+\frac{1}{2}, 0\right), & 0 \leqslant i \leqslant m-2,\end{cases} \\
& g(x)=\frac{1}{\sqrt{n}} R\left(\frac{\pi}{2}\right) \sigma_{y}(x)+\left(-m+\frac{1}{2}, 0\right),
\end{aligned}
$$

where $R(\theta)$ is the rotation through the angle $\theta$ and $\sigma_{y}$ denotes the reflection about the $y$-axis. Let $T$ be the $n$-repset defined by the IFS $\mathcal{F}:=\{g\} \cup\left\{f_{i}\right\}_{i=-m}^{m-2}$ (see Figure 1). We have the following theorem.

Theorem 1.1. Let $n=2 m$ with $m \geqslant 2$, and let $T$ be as defined above. Then $T$ is a connected $n$-reptile. Moreover, the interior of $T$ is the union of the largest component, together with countably many geometrically similar sets, with the closure of each of these components containing infinitely many holes.

Theorem 1.1 thus reduces the original question in [3] to whether there exist 2-reptiles or 3 -reptiles in the plane with holes. The proof of Theorem 1.1 will be given in $\S 3$.

Theorem 1.1 also answers the following questions raised in [10] in the affirmative: in the case that the interior of a reptile $T$ in $\mathbb{R}^{2}$ has infinitely many components, is it 
(a)

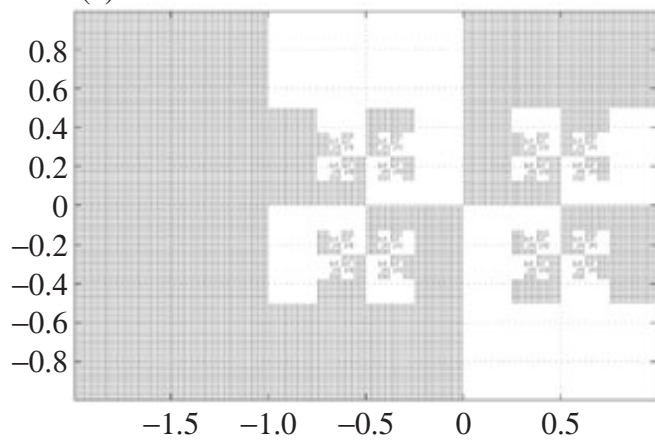

(b)

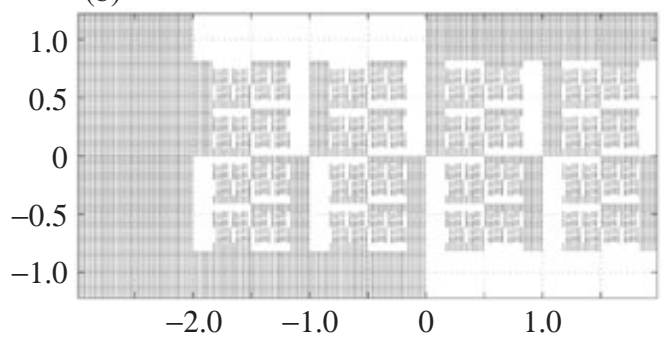

(c)

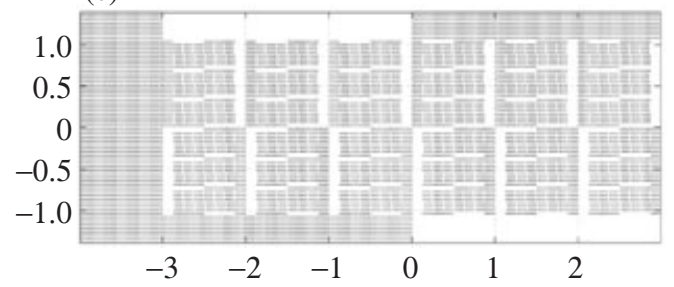

(d)

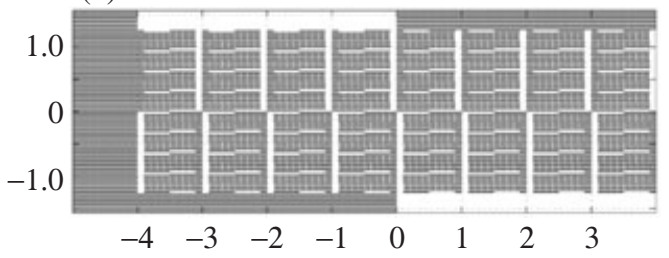

Figure 1. The first four reptiles in the family: (a) $F_{4}$, (b) $F_{6}$, (c) $F_{8}$, (d) $F_{10}$.

possible that the closure of some component is not a topological disc, and is it possible that the closure of every component of the interior of $T$ has a hole?

In $\S 2$ we develop a general method for constructing connected reptiles. To properly describe the method we will need the following notation, which will be used throughout this paper.

We let $\mu=\mu_{d}$ be the $d$-dimensional Lebesgue measure on $\mathbb{R}^{d}$. For $A, B \subseteq \mathbb{R}^{d}$, we denote by $A^{\circ}, \operatorname{cl}(A)$, and $\partial A$ the interior, closure and boundary of $A$, respectively, and say that $A$ and $B$ are essentially disjoint if $\mu(A \cap B)=0$. If $A$ is finite, we denote the cardinality of $A$ by $|A|$. We let id denote the identity map on $\mathbb{R}^{d}$. For a sequence of maps $g_{1}, \ldots, g_{k}$ on $\mathbb{R}^{d}$, we denote the composition $g_{1} \circ \cdots \circ g_{k}$ simply by $g_{1} \cdots g_{k}$. Let $\mathcal{R}, \mathcal{S}$ be two sets of mappings on $\mathbb{R}^{d}$ and $A \subseteq \mathbb{R}^{d}$; we use the following convenient notation:

$$
\mathcal{R S}:=\{r s: r \in \mathcal{R}, s \in \mathcal{S}\} \text { and } \mathcal{R}(A):=\bigcup_{r \in \mathcal{R}} r(A) .
$$

Let $\mathcal{F}=\left\{f_{i}\right\}_{i=1}^{n}$ be an IFS of $n$ similitudes on $\mathbb{R}^{d}$ with contraction ratio $1 / \sqrt[d]{n}$. Let $T_{0}$ be a non-empty compact subset of $\mathbb{R}^{d}$, invariant under $\mathcal{F}$ (i.e. $\mathcal{F}\left(T_{0}\right) \subseteq T_{0}$ ), such that

$$
T_{0}^{\circ} \neq \emptyset, \quad \operatorname{cl}\left(T_{0}^{\circ}\right)=T_{0} \quad \text { and } \quad \mu\left(T_{0}^{\circ}\right)=\mu\left(T_{0}\right) .
$$

Define

$$
T_{k}:=\mathcal{F}\left(T_{k-1}\right) \quad \text { for } k \geqslant 1 \text { and } T:=\bigcap_{k=0}^{\infty} T_{k} .
$$

Clearly, $T$ is the attractor of $\mathcal{F}$ and is an $n$-repset. 
Let $\mathcal{G}$ be a finite group of orthogonal transformations satisfying the following condition.

Condition 1.2. $r\left(T_{0}\right)=T_{0}$ for all $r \in \mathcal{G}, \mathcal{G}\left(T_{1}\right)=T_{0}$.

Define a subset $\mathcal{F}_{1}$ of $\mathcal{F}$ induced by $\mathcal{G}$ as

$$
\mathcal{F}_{1}=\mathcal{F}_{1}(\mathcal{F}, \mathcal{G}):=\{f \in \mathcal{F}: f \mathcal{G} \subseteq \mathcal{F}\},
$$

i.e. $f \in \mathcal{F}_{1}$ if and only if $f \mathcal{G} \subseteq \mathcal{F}$. Assume that $\mathcal{G}$ induces a partition of $\mathcal{F}$ into $\mathcal{F}_{1}$ and $\mathcal{F}_{2}:=\mathcal{F} \backslash \mathcal{F}_{1}$ such that the following condition holds.

Condition 1.3. $\mathcal{F}_{1} \neq \emptyset$ and, for all $r \in \mathcal{G}, r \mathcal{F}_{2}=\mathcal{F}_{2} r$ (i.e. $\left\{r f: f \in \mathcal{F}_{2}\right\}=$ $\left.\left\{f r: f \in \mathcal{F}_{2}\right\}\right)$.

It is easy to check that (1.6) and Condition 1.3 actually imply that

$$
\mathcal{F}_{1} \mathcal{G}=\mathcal{F}_{1} \quad \text { and } \quad \mathcal{G F}_{2}=\mathcal{F}_{2} \mathcal{G}
$$

Define

$$
\left.\begin{array}{c}
\mathcal{V}_{0}:=\left\{f\left(T_{0}\right): f \in \mathcal{F}_{1}\right\}, \quad \mathcal{V}_{k}:=\left\{f(U): U \in \mathcal{V}_{k-1} \text { and } f \in \mathcal{F}_{2}\right\} \quad \forall k \geqslant 1, \\
\mathcal{V}:=\bigcup_{k=0}^{\infty} \mathcal{V}_{k}, \quad V_{k}:=\bigcup_{S \in \mathcal{V}_{k}} S \quad \forall k \geqslant 0, \quad V:=\bigcup_{k=0}^{\infty} V_{k} .
\end{array}\right\}
$$

Note that if $\mathcal{F}_{2}=\emptyset$ then $\mathcal{V}_{k}=\emptyset$ for all $k \geqslant 1$. The following condition guarantees connectedness of $T$.

Condition 1.4. $T_{0}$ is connected and for some $k \geqslant 0$, some component of $\bigcup_{i=0}^{k} V_{i}$ has non-empty intersection with $f\left(T_{0}^{\circ}\right)$ for every $f \in \mathcal{F}$.

Theorem 1.5. Let $\mathcal{F}=\left\{f_{i}\right\}_{i=1}^{n}$ be an IFS with attractor $T$, let $T_{0}$ be invariant under $\mathcal{F}$ and satisfy (1.4), and let $\left\{T_{k}\right\}_{k=0}^{\infty}$ be described as in (1.5). Suppose there exists a finite group of orthogonal transformations $\mathcal{G}$ on $\mathbb{R}^{d}$ that satisfies Condition 1.2 and induces a partition $\left\{\mathcal{F}_{1}, \mathcal{F}_{2}\right\}$ of $\mathcal{F}$ satisfying Condition 1.3 above. Then the following hold.

(a) The attractor $T$ is an $n$-reptile.

(b) If, in addition, Condition 1.4 is satisfied, then $T$ is connected.

Theorem 1.5 provides a new method of constructing reptiles that cannot be obtained directly by the existing general method described by Bandt [1, Theorem 2]. In Bandt's result, the inverse of the linear part of each similitude in the IFS must be an integer matrix. This is not satisfied by some of the reptiles described in Theorem 1.1.

In $\S 4$, we use the method in $\S 2$ to construct reptiles with various interesting topological properties. In particular, we construct a connected reptile in $\mathbb{R}^{2}$ whose interior consists of infinitely many components, with the closure of some of them having holes and some of them being topological discs. We also construct a connected reptile in $\mathbb{R}^{2}$ whose interior consists of infinitely many components, with the closure of each component having finitely many holes. Lastly, we construct a connected piecewise polygonal 16-reptile with a hole. 
Although most reptiles constructed in this paper are defined by IFSs that involve reflections, it is worth pointing out that Example 4.2 is a reptile with a hole and is defined by an IFS without involving any reflection. The smallest $n$ for which we can construct such an $n$-reptile is 16 . All other reptiles with holes constructed in this paper are defined by IFSs that involve at least two distinct orthogonal transformations. We do not know whether there exist reptiles with holes that are defined by similitudes having a common orthogonal transformation.

The rest of this paper is organized as follows. In $\S 2$, we describe our construction of reptiles in $\mathbb{R}^{d}$ and prove Theorem 1.5 . In $\S 3$, we study the particular family of reptiles defined by (1.2) and (1.3) and prove Theorem 1.1. Lastly, in $\S 4$, we construct other reptiles with various topological properties.

\section{The construction}

Let $T_{0}$ be a non-empty compact subset of $\mathbb{R}^{2}$ satisfying the conditions in (1.4). Let $\mathcal{F}=\left\{f_{i}\right\}_{i=1}^{n}$ be an IFS of similitudes with contraction ratio $1 / \sqrt[d]{n}$, and thus similarity dimension $d$, such that $\mathcal{F}\left(T_{0}\right) \subseteq T_{0}$. Let $\left\{T_{k}\right\}_{k=0}^{\infty}$ be defined as in (1.5).

Lemma 2.1. $\left\{T_{k}\right\}_{k=0}^{\infty}$ is a $\subseteq$-decreasing sequence of compact sets.

Proof. Clearly, $T_{1} \subseteq T_{0}$. Suppose we have shown that $T_{k} \subseteq \cdots \subseteq T_{0}$ for some $k$. Now $T_{k+1}=\mathcal{F}\left(T_{k}\right) \subseteq \mathcal{F}\left(T_{k-1}\right)=T_{k}$. The compactness is obvious.

Define $T=\bigcap_{k=0}^{\infty} T_{k}$ as in (1.5). Then $T$ is the attractor of $\mathcal{F}$ and is an $n$-repset. Let $\mathcal{G}$ be a finite group of orthogonal transformations satisfying Condition 1.2, and assume that $\mathcal{G}$ induces a partition $\left\{\mathcal{F}_{1}, \mathcal{F}_{2}\right\}$ of $\mathcal{F}$ such that Condition 1.3 in $\S 1$ is satisfied. We will assume that $\left\{T_{k}\right\}_{k=0}^{\infty}, T, \mathcal{F}, \mathcal{G}, \mathcal{F}_{1} \neq \emptyset$ and $\mathcal{F}_{2}$ are fixed as above for the remainder of this section.

Proposition 2.2. Let $\left\{T_{k}\right\}_{k=0}^{\infty}, T, \mathcal{G}, \mathcal{F}, \mathcal{F}_{1}$ and $\mathcal{F}_{2}$ satisfy the hypotheses of Theorem 1.5. Then

(a) $\mathcal{G}(T)=T_{0}$ and $\mathcal{F}_{1}\left(T_{0}\right) \subseteq T$;

(b) $\mathcal{F}$ satisfies the open set condition and $T$ is an $n$-reptile.

Proof. We prove (a) by induction. Since $\left\{T_{k}\right\}_{k=0}^{\infty}$ is $\subseteq$-decreasing, it suffices to show that, for every integer $k \geqslant 0, \mathcal{G}\left(T_{k}\right)=T_{0}$ and $\mathcal{F}_{1}\left(T_{0}\right) \subseteq T_{k}$. This is obviously true for $k=0$ by assumption. Assume that it is true for some $k \geqslant 0$.

Suppose $f \in \mathcal{F}_{1}$. Since $f r \in \mathcal{F}$ for all $r \in \mathcal{G}$, we have

$$
T_{k+1} \supseteq \bigcup_{r \in \mathcal{G}} f r\left(T_{k}\right)=f\left(\mathcal{G}\left(T_{k}\right)\right)=f\left(T_{0}\right) .
$$

Thus, $\mathcal{F}_{1}\left(T_{0}\right) \subseteq T_{k+1}$.

It remains to prove that $\mathcal{G}\left(T_{k+1}\right)=T_{0}$. We have shown that $\mathcal{F}_{1}\left(T_{0}\right) \subseteq T_{k+1}$. So we get $\mathcal{G F}_{1}\left(T_{0}\right) \subseteq \mathcal{G}\left(T_{k+1}\right)$. Next, by (1.7) and the induction hypothesis,

$$
\mathcal{G F}_{2}\left(T_{0}\right)=\mathcal{F}_{2} \mathcal{G}\left(T_{0}\right)=\mathcal{F}_{2}\left(T_{0}\right)=\mathcal{F}_{2} \mathcal{G}\left(T_{k}\right)=\mathcal{G F}_{2}\left(T_{k}\right) \subseteq \mathcal{G}\left(T_{k+1}\right)
$$


Combining the two inclusions above, we get $\mathcal{G F}\left(T_{0}\right) \subseteq \mathcal{G}\left(T_{k+1}\right)$. This implies, by the definition $\mathcal{F}\left(T_{0}\right)=T_{1}$ and the assumption $\mathcal{G}\left(T_{1}\right)=T_{0}$ in Condition 1.2, that $T_{0} \subseteq \mathcal{G}\left(T_{k+1}\right)$. The reverse inclusion is obvious and this completes the induction.

To prove (b) we use the fact that, since $\mathcal{F}_{1} \neq \emptyset$, part (a) implies that $T^{\circ} \neq \emptyset$. Since the similarity dimension of $\mathcal{F}$ is $d$, it follows that $\mathcal{F}$ satisfies the open set condition [11] and hence $T$ is an $n$-reptile.

We need to study the structure of $\mathcal{F}_{1}$ in more detail. Define an equivalence relation ' $\sim$ ' on $\mathcal{F}_{1}$ by

$$
f \sim g \text { if and only if } f \in g \mathcal{G} .
$$

Since $\mathcal{G}$ is a group, it is easy to see that ' $\sim$ ' is indeed an equivalence relation. Denote the equivalence class of $f \in \mathcal{F}_{1}$ by $[f]$. Throughout the rest of this section, we will fix a complete set of representatives $\mathcal{F}_{1}^{*}=\left\{f_{1}, \ldots, f_{q}\right\}$ for the quotient set $\mathcal{F}_{1} / \sim$.

Lemma 2.3. Assume the same hypotheses as in Theorem 1.5. Let $\left\{\mathcal{V}_{k}\right\}_{k=0}^{\infty}$ be defined as in (1.8), and let $\mathcal{F}_{1}^{*}:=\left\{f_{1}, \ldots, f_{q}\right\}$ be a complete set of representatives for the quotient set $\mathcal{F}_{1} / \sim$. Then

(a) $\left|\mathcal{V}_{0}\right|=\left|\mathcal{F}_{1}^{*}\right|=\left|\mathcal{F}_{1}\right| /|\mathcal{G}|$;

(b) $\left|\mathcal{G F}_{1}^{*}\right|=\left|\mathcal{F}_{1}\right|$;

(c) $\mathcal{G F}_{1}^{*}\left(T_{0}\right)=\mathcal{G F}_{1}\left(T_{0}\right)$.

Proof. (a) Let $f \in \mathcal{F}_{1}$ and assume that $f \in\left[f_{1}\right]$, say. Then $f=f_{1} r$ for some $r \in \mathcal{G}$, which implies that $f\left(T_{0}\right)=f_{1} r\left(T_{0}\right)=f_{1}\left(T_{0}\right)$. Hence, $\left|\mathcal{V}_{0}\right| \leqslant\left|\mathcal{F}_{1}^{*}\right|$.

On the other hand, let $f, g \in \mathcal{F}_{1}$ satisfy $f\left(T_{0}\right)=g\left(T_{0}\right)$. Then by Proposition 2.2(a), $f \mathcal{G}(T)=g \mathcal{G}(T)$. The open set condition implies that $f=g r$ for some $r \in \mathcal{G}$. Hence, $f \sim g$. It follows that $\left|\mathcal{V}_{0}\right| \geqslant\left|\mathcal{F}_{1}^{*}\right|$.

To prove the last equality in (a), it suffices to show that, for all $f^{*}, g^{*} \in \mathcal{F}_{1}^{*}$, $\left|\left[f^{*}\right]\right|=\left|\left[g^{*}\right]\right|$. This can be verified directly by showing that the mapping $\tau:\left[f^{*}\right] \rightarrow\left[g^{*}\right]$ defined by $\tau\left(f^{*} r\right)=g^{*} r, r \in \mathcal{G}$, is a bijection.

(b) Define a mapping $\sigma: \mathcal{G F}_{1}^{*} \rightarrow \mathcal{F}_{1}$ by

$$
\sigma(r f)=f r \quad \text { for all }(r, f) \in \mathcal{G} \times \mathcal{F}_{1}^{*} .
$$

It is direct to check that $\sigma$ is a bijection and thus (b) follows.

(c) It suffices to show that $\mathcal{G F}_{1}^{*}\left(T_{0}\right) \supseteq \mathcal{G F}_{1}\left(T_{0}\right)$; the reverse inclusion is obvious. Let $r \in \mathcal{G}$ and $f \in \mathcal{F}_{1}$. Then there exists $f^{*} \in \mathcal{F}_{1}^{*}$ and $s \in \mathcal{G}$ such that $f=f^{*} s$. Thus,

$$
r f\left(T_{0}\right)=r f^{*} s\left(T_{0}\right)=r f^{*}\left(T_{0}\right) \subseteq \mathcal{G} \mathcal{F}_{1}^{*}\left(T_{0}\right),
$$

completing the proof.

Proposition 2.4. Assume the same hypotheses as in Theorem 1.5. Let $\left\{\mathcal{V}_{k}\right\}_{k=0}^{\infty}, \mathcal{V}$, $\left\{V_{k}\right\}_{k=0}^{\infty}$, and let $V$ be defined as in (1.8). Then

(a) $\mathcal{V}$ is a mutually essentially disjoint collection; 
(b) $V \subseteq T$ and $\mu(V)=\mu(T)$;

(c) $\{r(T)\}_{r \in \mathcal{G}}$ is a mutually essentially disjoint collection;

(d) $\mathcal{G} \neq\{$ id $\}$ if and only if $T \neq T_{0}$;

(e) $T_{0}$ is an $n$-reptile.

Proof. To prove (a), it suffices to prove by induction on $k$ the claim that any set in $\mathcal{V}_{k}$ is essentially disjoint from any distinct set in $\mathcal{V}_{j}, j \geqslant 0$.

We first consider the case $k=j=0$. Let $R, S \in \mathcal{V}_{0}$ be distinct. Then there exist distinct $f, g \in \mathcal{F}_{1}$ such that $R=f\left(T_{0}\right)$ and $S=g\left(T_{0}\right)$. Note that, for any $r, s \in \mathcal{G}$, $f r \neq g s$; otherwise, we would have $R=f\left(T_{0}\right)=f r\left(T_{0}\right)=g s\left(T_{0}\right)=g\left(T_{0}\right)=S$. By Proposition 2.2,

$$
f\left(T_{0}\right)=\bigcup_{r \in \mathcal{G}} f r(T) \quad \text { and } \quad g\left(T_{0}\right)=\bigcup_{s \in \mathcal{G}} g s(T) .
$$

Now the open set condition implies that $g\left(T_{0}\right)$ and $f\left(T_{0}\right)$ are essentially disjoint. So any two distinct sets in $\mathcal{V}_{0}$ are essentially disjoint.

Now let $j>0$. Suppose $R \in \mathcal{V}_{0}$ and $S \in \mathcal{V}_{j}$. Let $S^{*} \in \mathcal{V}_{j-1}$ and $f \in \mathcal{F}_{2}$ be such that $f\left(S^{*}\right)=S$. Since $S^{*} \subseteq T$ and $\mathcal{F}$ satisfies the open set condition, $S$ is essentially disjoint from $R$. Thus the claim holds for $k=0$. Assume that it holds for some $k \geqslant 0$.

Let $R \in \mathcal{V}_{k+1}$ and $S \in \mathcal{V}_{j}$ for some $j \geqslant 0$. If $j=0$, then the above proof already implies that $R$ and $S$ are essentially disjoint. So we assume $j>0$. Let $R^{*} \in \mathcal{V}_{k}, S^{*} \in \mathcal{V}_{j-1}$ and $f, g \in \mathcal{F}_{2}$ be such that $f\left(R^{*}\right)=R$ and $g\left(S^{*}\right)=S$. If $f \neq g$, then the open set condition and the fact that $R^{*}, S^{*} \subseteq T$ yield that $f\left(R^{*}\right)$ and $g\left(S^{*}\right)$ are essentially disjoint. If $f=g$, then $R^{*} \neq S^{*}$. So, by the induction hypothesis, $R^{*}$ and $S^{*}$ are essentially disjoint. Thus, $f\left(R^{*}\right)$ and $g\left(S^{*}\right)$ are essentially disjoint. This completes the proof of the claim and (a) follows.

We now prove (b) and (c). The fact that $T \subseteq V$ follows directly from Proposition 2.2(a) and the construction of $V$. Let $q=\left|\mathcal{V}_{0}\right|$ and $m=|\mathcal{G}|$. By Lemma 2.3(a), $\left|\mathcal{F}_{1}\right|=m q$, and therefore $\left|\mathcal{F}_{2}\right|=n-m q$. Using (a) above, we find that

$$
\mu(V)=\sum_{k=0}^{\infty} \mu\left(V_{k}\right)=\sum_{k=0}^{\infty} \mu\left(T_{0}\right) \frac{q}{n}\left(\frac{n-m q}{n}\right)^{k}=\frac{\mu\left(T_{0}\right)}{m} .
$$

Since $\mathcal{G}(T)=T_{0}$, it now follows that $\{r(T)\}_{r \in \mathcal{G}}$ is an essentially disjoint collection and that $\mu(V)=\mu(T)$.

To prove $(\mathrm{d})$, note that when $\mathcal{G}=\{\mathrm{id}\}$, the relation $\mathcal{G}(T)=T_{0}$ implies that $T=T_{0}$. If $\mathcal{G} \neq\{$ id $\}$, then $m=|\mathcal{G}|>1$. The proof of (b) and (c) above yields $\mu\left(T_{0}\right)=m \mu(T)$. Thus, $T \neq T_{0}$. 
To prove (e), note that if $\mathcal{G}=\{\mathrm{id}\}$, then $T_{0}=T$. So we may assume that $\mathcal{G} \neq\{\mathrm{id}\}$. By Proposition 2.2, Lemma 2.3 and (1.7), we find that

$$
\begin{aligned}
& T_{0}=\mathcal{G}(T) \\
& =\mathcal{G F}_{1}(T) \cup \mathcal{G F}_{2}(T) \\
& =\mathcal{G F}_{1} \mathcal{G}(T) \cup \mathcal{F}_{2} \mathcal{G}(T) \quad \text { (by (1.7)) } \\
& =\mathcal{G F}_{1}\left(T_{0}\right) \cup \mathcal{F}_{2}\left(T_{0}\right) \quad \text { (by Proposition 2.2(a)) } \\
& =\mathcal{G F}_{1}^{*}\left(T_{0}\right) \cup \mathcal{F}_{2}\left(T_{0}\right) \quad \text { (by Lemma 2.3(c)). }
\end{aligned}
$$

Thus, $T_{0}$ is the attractor of $\mathcal{G F}_{1}^{*} \cup \mathcal{F}_{2}$. By (c), $\{r(T)\}_{r \in \mathcal{G}}$ is a mutually essentially disjoint collection. Hence, $\mathcal{G F}_{1}^{*}\left(T_{0}\right)=\mathcal{G F}_{1}(T)$ and $\mathcal{F}_{2}\left(T_{0}\right)=\mathcal{G F}_{2}(T)$ are essentially disjoint, and thus $\mathcal{G F}_{1}^{*} \cap \mathcal{F}_{2}=\emptyset$. Now, Lemma 2.3(b) implies that $\left|\mathcal{G F}_{1}^{*} \cup \mathcal{F}_{2}\right|=|\mathcal{F}|=n$. Since $T_{0}^{\circ} \neq \emptyset, T_{0}$ is an $n$-reptile.

We will now strengthen Proposition 2.4(c) to $T^{\circ} \subseteq V$ and prove Theorem 1.5(b). We need two lemmas.

Lemma 2.5. Assume the same hypotheses as in Theorem 1.5. Then, for any subset $\left\{h_{1}, \ldots, h_{k}\right\} \subseteq \mathcal{F}_{2}$,

$$
\operatorname{cl}\left(\left(T \cap h_{1} \cdots h_{k}\left(T_{0}\right)\right)^{\circ}\right)=h_{1} \cdots h_{k}(T) .
$$

Proof. Fix $r \in \mathcal{G} \backslash\{$ id $\}$. Since $h_{1} \in \mathcal{F}_{2}, h_{1} r(T) \subseteq r(T)$. So, $h_{1} r(T)$ is essentially disjoint from both $T$ and $h_{1}(T)$. By Proposition 2.2(a),

$$
\begin{aligned}
\left(T \cap h_{1} \cdots h_{k}\left(T_{0}\right)\right)^{\circ} & \subseteq\left(T \cap\left(h_{1} \cdots h_{k}(T) \cup \bigcup_{r \in \mathcal{G} \backslash\{\text { id }\}} h_{1} r(T)\right)\right)^{\circ} \\
& \subseteq\left(\left(T \cap h_{1} \cdots h_{k}(T)\right) \cup \partial T\right)^{\circ} \\
& =\left(h_{1} \cdots h_{k}(T) \cup \partial T\right)^{\circ} \\
& \subseteq h_{1} \cdots h_{k}(T) .
\end{aligned}
$$

Hence, $\operatorname{cl}\left(\left(T \cap h_{1} \cdots h_{k}\left(T_{0}\right)\right)^{\circ}\right) \subseteq h_{1} \cdots h_{k}(T)$. The reverse inequality is straightforward and this completes the proof.

Lemma 2.6. Assume the same hypotheses as in Theorem 1.5. Suppose $h_{1}, \ldots, h_{k} \in \mathcal{F}$ and $U:=h_{1} \cdots h_{k}\left(T_{0}\right)$ is not a subset of $V$. Then

$$
\operatorname{cl}\left(\left(T \cap h_{1} \cdots h_{k}\left(T_{0}\right)\right)^{\circ}\right)=h_{1} \cdots h_{k}(T) .
$$

Proof. By Lemma 2.5 it is enough to show that $h_{i} \in \mathcal{F}_{2}$ for every $1 \leqslant i \leqslant k$. Note that $h_{1} \in \mathcal{F}_{2}$; otherwise $U \subseteq h_{1}\left(T_{0}\right) \in \mathcal{V}_{0}$. Assume now that $1<i \leqslant k$ and that we have shown $h_{1}, \ldots, h_{i-1} \in \mathcal{F}_{2}$. If $h_{i} \in \mathcal{F}_{1}$, then $h_{i}\left(T_{0}\right) \in \mathcal{V}_{0}$. So, $h_{1} \cdots h_{k}\left(T_{0}\right) \subseteq h_{1} \cdots h_{i}\left(T_{0}\right) \in \mathcal{V}_{i}$, a contradiction. Thus, $h_{i} \in \mathcal{F}_{2}$ and the result follows.

Proposition 2.7. Under the hypotheses of Theorem 1.5, $T^{\circ} \subseteq V$. 
Proof. If $\mathcal{G}=\{\mathrm{id}\}$, then $T=T_{0}=V$ and we are done. So we assume that $\mathcal{G} \neq\{\mathrm{id}\}$ and thus $T \neq T_{0}$ by Proposition 2.4(d). Let $x \in T \backslash V$, let $B$ be an open ball centred at $x$, and let $h_{1}, \ldots, h_{k} \in \mathcal{F}$ such that $x \in h_{1} \cdots h_{k}\left(T_{0}\right) \subseteq B$. We claim that $B \nsubseteq T$. Suppose $B \subseteq T$. We note that since $x \notin V, h_{1} \cdots h_{j}\left(T_{0}\right) \nsubseteq V$. So, by Lemma 2.6,

$$
h_{1} \cdots h_{j}\left(T_{0}\right)=\operatorname{cl}\left(\left(T \cap h_{1} \cdots h_{j}\left(T_{0}\right)\right)^{\circ}\right)=h_{1} \cdots h_{j}(T),
$$

This contradicts $T \neq T_{0}$ and the claim is proved. Since $B$ was arbitrary, $x \notin T^{\circ}$ and the proposition follows.

Lemma 2.8. Assume the same hypotheses as in Theorem 1.5 and assume in addition that Condition 1.4 holds. Then $T$ is connected.

Proof. Let $W$ be a component of $\bigcup_{i=0}^{k} V_{i}$ that has non-empty intersection with $f\left(T_{0}^{\circ}\right)$ for every $f \in \mathcal{F}$. Then Proposition 2.4(b) implies that $W \subseteq T$. By a result of Hata $[6$, Theorem 4.6], it is enough to show that $W \cap f(T) \neq \emptyset$ for every $f \in \mathcal{F}$.

First, let $f \in \mathcal{F}_{1}$. Since $f\left(T_{0}\right)$ is connected and $W \cap f\left(T_{0}\right) \neq \emptyset$, we have $f\left(T_{0}\right) \subseteq W$. Thus, $W \cap f(T) \neq \emptyset$.

Now assume that $\mathcal{F}_{2} \neq \emptyset$ and let $f \in \mathcal{F}_{2}$. Since $W \cap f\left(T_{0}^{\circ}\right) \neq \emptyset$ and $W=\operatorname{cl}\left(W^{\circ}\right)$, $\left(W \cap f\left(T_{0}\right)\right)^{\circ} \neq \emptyset$. It now follows by using Lemma 2.5 that

$$
\left(W \cap f\left(T_{0}\right)\right)^{\circ} \subseteq \operatorname{cl}\left(\left(W \cap f\left(T_{0}\right)\right)^{\circ}\right) \subseteq \operatorname{cl}\left(\left(T \cap f\left(T_{0}\right)\right)^{\circ}\right) \subseteq f(T) .
$$

Thus, $W \cap f(T) \neq \emptyset$. This completes the proof.

Theorem 1.5 now follows by combining Propositions 2.2 and Lemma 2.8.

\section{A family of planar reptiles with holes}

Let $n=2 m$ be an even integer greater than or equal to 4 . Let $T_{0}:=[-m, m] \times$ $[-\sqrt{m / 2}, \sqrt{m / 2}]$. Clearly, $T_{0}$ satisfies the conditions in (1.4). Let $f_{i},-m \leqslant i \leqslant m-2$, and let $g$ be defined as in (1.2) and (1.3), respectively. Let

$$
\mathcal{F}:=\{g\} \cup\left\{f_{i}\right\}_{i=-m}^{m-2} .
$$

Use these $T_{0}$ and $\mathcal{F}$ to define $\left\{T_{k}\right\}_{k=0}^{\infty}$ as in (1.5), and let $T=\bigcap_{k=0}^{\infty} T_{k}$ be the attractor of $\mathcal{F}$ (see Figure 1). Let

$$
Q_{0}=[-m,-m+1] \times[-\sqrt{m / 2}, \sqrt{m / 2}] .
$$

Let $\mathcal{G}:=\left\{\mathrm{id}, \sigma_{y}\right\}$. Then $\mathcal{G}$ is a finite group of orthogonal transformations. Let $\mathcal{F}_{1}$ be the subset of $\mathcal{F}$ induced by $\mathcal{G}$ as defined in (1.6) and let $\mathcal{F}_{2}=\mathcal{F} \backslash \mathcal{F}_{1}$.

Lemma 3.1. Let $\left\{T_{k}\right\}_{k=0}^{\infty}, T, \mathcal{F}, \mathcal{G}, \mathcal{F}_{1}$ and $\mathcal{F}_{2}$ be defined as above. Then

(a) $r\left(T_{0}\right)=T_{0}$ for all $r \in \mathcal{G}$ and $\mathcal{G}\left(T_{1}\right)=T_{0}$;

(b) $\mathcal{F}_{1}=\left\{f_{-m}, g\right\}$; 
(c) $r \mathcal{F}_{2}=\mathcal{F}_{2} r$ for all $r \in \mathcal{G}$.

Proof. Proof of (a) follows by a direct verification. To prove (b) we note that $f_{-m} \sigma_{y}=$ $g$ and thus $g \sigma_{y}=f_{-m}$. Moreover, for any $f \in \mathcal{F} \backslash\left\{f_{-m}, g\right\}, f \sigma_{y} \notin \mathcal{F}$. Proof of (c) follows by showing that $\sigma_{y} f_{i}=f_{-i-1} \sigma_{y}$ for $-m+1 \leqslant i \leqslant m-2$.

Lemma 3.1 shows that both Conditions 1.2 and 1.3 in $\S 1$ hold. Thus, $\mathcal{F},\left\{T_{k}\right\}_{k=0}^{\infty}, T$, $\mathcal{G}, \mathcal{F}_{1}$ and $F_{2}$ satisfy all hypotheses of Theorem 1.5. Note that $Q_{0}=f_{-m}\left(T_{0}\right)$. So, by Proposition 2.2, we have the following lemma.

Lemma 3.2. $T$ is an n-reptile, $T \cup \sigma_{y}(T)=T_{0}$ and $Q_{0} \subseteq T$.

For the rest of this section, let $\left\{\mathcal{V}_{k}\right\}_{k=0}^{\infty}, \mathcal{V},\left\{V_{k}\right\}_{k=0}^{\infty}$ and $V$ be defined as in (1.8).

Lemma 3.3. Condition 1.4 holds and thus $T$ is connected.

Proof. $T_{0}$ is obviously connected. By examining $f\left(T_{0}^{\circ}\right)$ for all $f \in \mathcal{F}$, it suffices to prove that $W=V_{0} \cup V_{1} \cup V_{2}$ is connected. This may be done by a direct verification.

To describe the components of $T^{\circ}$, we define three additional sets of maps as follows:

$$
\mathcal{H}_{1}:=\left\{f_{i}\right\}_{i=-m+1}^{-1}, \quad \mathcal{H}_{2}:=\left\{f_{i}\right\}_{i=0}^{m-2}, \quad \mathcal{H}_{3}:=f_{-m+1} \mathcal{H}_{2}=\left\{f_{-m+1} f_{i}\right\}_{i=0}^{m-2} .
$$

Note that $\mathcal{H}_{1} \cup \mathcal{H}_{2}=\mathcal{F}_{2}$. We also define a sequence of subsets $Q_{k} \subseteq T, k \geqslant 1$, recursively as follows:

$$
Q_{1}:=\mathcal{H}_{1}\left(Q_{0}\right) \quad \text { and } \quad Q_{k}:=\mathcal{H}_{1}\left(Q_{k-1}\right) \cup \mathcal{H}_{3}\left(Q_{k-2}\right), \quad k \geqslant 2 .
$$

Let

$$
Q:=\operatorname{cl}\left(\bigcup_{k=0}^{\infty} Q_{k}\right)
$$

Note that $\bigcup_{k=0}^{\infty} Q_{k}^{\circ}$ is dense in $\bigcup_{k=0}^{\infty} Q_{k}$, so $Q=\operatorname{cl}\left(Q^{\circ}\right)$. We will show that $Q$ is the closure of the largest component of $T^{\circ}$.

Lemma 3.4. Let $\left\{Q_{k}\right\}_{k=0}^{\infty}$ be defined as above. Then

(a) for all $k \geqslant 0, Q_{k} \subseteq T \cap\left\{(x, y) \subseteq \mathbb{R}^{2}: x \leqslant 0\right\}$;

(b) $\mathcal{H}_{1}\left(Q^{\circ}\right) \cap \mathcal{H}_{3}\left(Q^{\circ}\right)=\emptyset$;

(c) the $Q_{k}$ are mutually essentially disjoint;

(d) $Q^{\circ}$ is connected.

Proof. (a) By Lemma 3.2, $Q_{0} \cup Q_{1} \subseteq T \cap\left\{(x, y) \subseteq \mathbb{R}^{2}: x \leqslant 0\right\}$. The assertion follows by using induction and the invariance of $T$ under the $f_{i}$.

(b) By using part (a) and the definitions of $\mathcal{H}_{1}$ and $\mathcal{H}_{3}$, we see that

$$
\mathcal{H}_{1}\left(Q^{\circ}\right) \subseteq\left\{(x, y) \in \mathbb{R}^{2}: y<0\right\}, \quad \mathcal{H}_{3}\left(Q^{\circ}\right) \subseteq\left\{(x, y) \in \mathbb{R}^{2}: y>0\right\},
$$

and the result follows. 
(c) Obviously, $Q_{0}$ and $Q_{1}$ are essentially disjoint. Assume that, for some $k \geqslant 1$, $Q_{0}, Q_{1}, \ldots, Q_{k}$ are essentially disjoint. Then

$$
Q_{k+1}^{\circ} \cap Q_{0}^{\circ}=\left[\mathcal{H}_{1}\left(Q_{k}^{\circ}\right) \cup \mathcal{H}_{3}\left(Q_{k-1}^{\circ}\right)\right] \cap Q_{0}^{\circ} \subseteq\left[\mathcal{H}_{1}\left(T^{\circ}\right) \cup \mathcal{H}_{3}\left(T^{\circ}\right)\right] \cap Q_{0}^{\circ}=\emptyset .
$$

By using the induction hypothesis and part (b), we have

$$
\begin{aligned}
Q_{k+1}^{\circ} \cap Q_{1}^{\circ} & =\left[\mathcal{H}_{1}\left(Q_{k}^{\circ}\right) \cup \mathcal{H}_{3}\left(Q_{k-1}^{\circ}\right)\right] \cap \mathcal{H}_{1}\left(Q_{0}^{\circ}\right) \\
& =\mathcal{H}_{1}\left(Q_{k}^{\circ} \cap Q_{0}^{\circ}\right) \cup\left[\mathcal{H}_{3}\left(Q_{k-1}^{\circ}\right) \cap \mathcal{H}_{1}\left(Q_{0}^{\circ}\right)\right] \\
& =\emptyset .
\end{aligned}
$$

Using a similar argument, for $2 \leqslant i \leqslant k$, we have

$$
\begin{aligned}
Q_{k+1}^{\circ} \cap Q_{i}^{\circ} & =\left[\mathcal{H}_{1}\left(Q_{k}^{\circ}\right) \cup \mathcal{H}_{3}\left(Q_{k-1}^{\circ}\right)\right] \cap\left[\mathcal{H}_{1}\left(Q_{i-1}^{\circ}\right) \cup \mathcal{H}_{3}\left(Q_{i-2}^{\circ}\right)\right] \\
& =\mathcal{H}_{1}\left(Q_{k}^{\circ} \cap Q_{i-1}^{\circ}\right) \cup\left[\mathcal{H}_{1}\left(Q_{k}^{\circ}\right) \cap \mathcal{H}_{3}\left(Q_{i-2}^{\circ}\right)\right] \\
& =\emptyset\left[\mathcal{H}_{3}\left(Q_{k-1}^{\circ}\right) \cap \mathcal{H}_{1}\left(Q_{i-1}^{\circ}\right)\right] \cup \mathcal{H}_{3}\left(Q_{k-1}^{\circ} \cap Q_{i-2}^{\circ}\right) \\
& \emptyset
\end{aligned}
$$

This proves part (c).

(d) Let $S_{k}:=\bigcup_{j=0}^{k} Q_{j}$. Since $\left\{S_{k}\right\}_{k=0}^{\infty}$ is a $\subseteq$-increasing sequence of sets, it is enough to show that the interior of $S_{k}$ is connected for every integer $k \geqslant 0$. We proceed by induction.

Clearly, $S_{0}=Q_{0}$ has a connected interior. When $k=1$, note that the left-hand edge of $f_{-m+1}\left(Q_{0}\right)$ is contained in the right-hand edge of $Q_{0}$ and the left-hand edge of $f_{i}\left(Q_{0}\right)$ is the right-hand edge of $f_{i-1}\left(Q_{0}\right)$ for $-m+2 \leqslant i \leqslant-1$. Thus, $S_{1}$ has a connected interior. Also, the left-hand edge of $\bigcup_{i=0}^{m-2} f_{-m+1} f_{i}\left(Q_{0}\right)$ is contained in the right-hand edge of $Q_{0}$, so $Q_{0} \cup \mathcal{H}_{3}\left(Q_{0}\right)$ has a connected interior. A similar argument actually proves the inductive step. We omit the details. This proves part (d).

For any index $J=\left(j_{1}, \ldots, j_{k}\right), 1 \leqslant j_{i} \leqslant n$, let $|J|=k$ denote the length of $J$. For any subset $A \subseteq \mathbb{R}^{2}$, let $f_{J}(A):=f_{j_{1}} \ldots f_{j_{k}}(A)$. If $J=\emptyset$, we interpret $f_{J}$ as the identity function. Given an index $J=\left(j_{1}, \ldots, j_{k}\right)$ and a symbol $j$ we define $j \oplus J=\left(j, j_{1} \ldots, j_{k}\right)$.

We define a sequence of collections of indices $\left\{\mathcal{I}_{k}\right\}_{k=0}^{\infty}$ recursively as follows:

$$
\begin{aligned}
& \mathcal{I}_{0}:=\{\emptyset\}, \\
& \mathcal{I}_{1}:=\{(i):-m+2 \leqslant i \leqslant m-2\}, \\
& \mathcal{I}_{k}:=\left\{i \oplus J: J \in \mathcal{I}_{k-1} \text { and }-m+1 \leqslant i \leqslant m-2\right\}, \quad k \geqslant 2 .
\end{aligned}
$$

Define $\mathcal{I}:=\bigcup_{k=0}^{\infty} \mathcal{I}_{k}$. For $A \subseteq \mathbb{R}^{2}$ and $k \geqslant 0$ we define

$$
\mathcal{I}_{k}(A):=\left\{f_{J}(A): J \in \mathcal{I}_{k}\right\} \quad \text { and } \quad \mathcal{I}(A):=\bigcup_{k=0}^{\infty} \mathcal{I}_{k}(A) .
$$

Let $Q^{*}:=\mathcal{H}_{2}(Q)$. Our next goal is to prove the following proposition, which describes all components of $T^{\circ}$. 
Proposition 3.5. The components of $T^{\circ}$ are $\left\{Q^{\circ}\right\} \cup \mathcal{I}\left(\left(Q^{*}\right)^{\circ}\right)$. Moreover, for distinct $I, J \in \mathcal{I},\left|f_{I}\left(Q^{*}\right) \cap f_{J}\left(Q^{*}\right)\right| \leqslant 2$.

Let $D:=\{(0,0),(0, \sqrt{m / 2})\}$ and $E:=\mathcal{H}_{2}(T)$. Define sequences of ordered pairs of subsets of $T$ as

$$
\mathcal{R}_{k}:=\left\{\left(f_{J}(E), f_{J}(D)\right): J \in \mathcal{I}_{k}\right\}, \quad k \geqslant 0, \text { and let } \mathcal{R}:=\bigcup_{k=0}^{\infty} \mathcal{R}_{k} .
$$

A simple inductive argument shows that $K \subseteq H$ for every $(H, K) \in \mathcal{R}$.

Given $A, B, X, Y \subseteq \mathbb{R}^{2}$ we say that $X$ separates $A$ from $B$ in $Y$ provided that, for every connected $C \subseteq Y$, if $C \cap A \neq \emptyset$ and $C \cap B \neq \emptyset$, then $C \cap X \neq \emptyset$.

Lemma 3.6. If $(H, K) \in \mathcal{R}$, then $K$ separates $H$ from $T \backslash H$ in $T$.

Proof. We leave it to the readers to convince themselves that the lemma holds for all pairs from steps $\mathcal{R}_{0}$ and $\mathcal{R}_{1}$ of the construction, and that for every $H \in \mathcal{I}_{1}(E)$ and $f \in \mathcal{F}_{2}$ we have $f(H) \subseteq\left(f\left(T_{0}\right)\right)^{\circ}$. Suppose now that $k>1$. Assume that the lemma holds for all pairs in $\mathcal{R}_{k-1}$ and that for every $H \in \mathcal{I}_{k-1}(E)$ and $f \in \mathcal{F}_{2}$ we have $f(H) \subseteq\left(f\left(T_{0}\right)\right)^{\circ}$.

Let $\left(H^{*}, K^{*}\right) \in \mathcal{R}_{k}$. Then there exist $(H, K) \in \mathcal{R}_{k-1}$ and $f \in \mathcal{F}_{2}$ such that $f(K)=K^{*}$ and $f(H)=H^{*}$. By assumption, $K$ separates $H$ from $T \backslash H$ and $H^{*} \subseteq\left(f\left(T_{0}\right)\right)^{\circ}$. So, $K^{*}$ separates $H^{*}$ from $f(T) \backslash H^{*}$ in $f(T)$. By Lemma $2.5, f(T)=\operatorname{cl}\left(\left(T \cap f\left(T_{0}\right)\right)^{\circ}\right)$. So, $K^{*}$ separates $H^{*}$ from $\operatorname{cl}\left(\left(T \cap f\left(T_{0}\right)\right)^{\circ}\right) \backslash H^{*}$. Since $H^{*} \subseteq\left(f\left(T_{0}\right)\right)^{\circ}, K^{*}$ separates $H^{*}$ from $T \backslash H^{*}$. Moreover, for all $g \in \mathcal{F}_{2}, g\left(H^{*}\right) \subseteq g\left(\left(f\left(T_{0}\right)\right)^{\circ}\right) \subseteq\left(g\left(T_{0}\right)\right)^{\circ}$, completing the induction.

Lemma 3.7. If $p \in T^{\circ} \backslash Q$, then there exists $(H, K) \in \mathcal{R}$ such that $p \in H$ and $Q^{\circ} \subseteq T \backslash H$.

Proof. By Proposition 2.7, $T^{\circ} \subseteq V$. So, there is some $k \geqslant 0$ such that $p \in V_{k}$. We use induction on $k$. Again we leave the easier cases of $k=0$ and $k=1$ to the reader.

Assume that $k>1$ and suppose that the lemma holds for $p \in V_{k-1}$. Let $p \in V_{k} \backslash Q$ and $f \in \mathcal{F}_{2}$ be such that $f^{-1}(p) \in V_{k-1}$.

If $f \in \mathcal{H}_{1}$, then $f^{-1}(p) \notin Q$ by the definition of $Q$. Note that $f^{-1}(p) \in T^{\circ}$. By induction hypothesis, there exists $(H, K) \in \mathcal{R}$ such that $f^{-1}(p) \in H$ and $Q^{\circ} \subseteq T \backslash H$. Since $(f(H), f(K)) \in \mathcal{R}, f(K)$ separates $f(H)$ from $T \backslash f(H)$. Note that $f\left(Q^{\circ}\right) \subseteq$ $f(T) \backslash f(H) \subseteq T \backslash f(H)$. Since $f(Q) \subseteq Q$ and $Q^{\circ}$ is connected and $f(K)$ is finite, we have $Q^{\circ} \subseteq T \backslash f(H)$. Also, $f^{-1}(p) \in H$ implies that $p \in f(H)$.

If $f \in \mathcal{H}_{2}$, then $p \in E$. Since $(E, D) \in \mathcal{R}$ and $Q^{\circ} \subseteq T \backslash E$, the assertion follows.

Lemma 3.8. $Q^{\circ}$ is a component of $T^{\circ}$ and for any $p \in T^{\circ} \backslash Q^{\circ}$ there exists $(H, K) \in \mathcal{R}$ such that $p \in H$ and $Q^{\circ} \subseteq T \backslash H$.

Proof. By Lemma 3.4(d), $Q^{\circ}$ is connected.

By way of contradiction, suppose that $Q^{\circ}$ is a not a maximal connected set in $T^{\circ}$. Let $N$ denote the component of $T^{\circ}$ containing $Q^{\circ}$. Since $Q=\operatorname{cl}\left(Q^{\circ}\right)$, there is a $p \in N \backslash Q$. By Lemma 3.7, there is an ordered pair $(H, K) \in \mathcal{R}$ such that $p \in H$ and $Q^{\circ} \subseteq T \backslash H$. 
In particular, $K$ is a finite set separating $Q^{\circ}$ from $p$. Thus, $p \notin N$, a contradiction. So, $Q^{\circ}$ is a component of $T^{\circ}$.

Let $p \in T^{\circ} \backslash Q^{\circ}$. Since $Q^{\circ}$ is a component of $T^{\circ}$ and $\operatorname{cl}\left(Q^{\circ}\right)=Q, p \notin Q$. By Lemma 3.7, there exists a $(H, K) \in \mathcal{R}$ such that $p \in H$ and $Q^{\circ} \subseteq T \backslash H$.

Lemma 3.9. Let $S \in \mathcal{I}_{k}\left(\left(Q^{*}\right)^{\circ}\right)$. Then $S$ is a component of $T^{\circ}$ and for any $p \in T^{\circ} \backslash S$ there is $(H, K) \in \bigcup_{l=k}^{\infty} \mathcal{R}_{k}$ such that $p \in H$ and $S \subseteq T \backslash H$. In particular, the closures of any two components of $T^{\circ}$ meet in at most two points.

Proof. We proceed by induction on $k$. The cases $k=0$ and $k=1$ can be verified directly by Lemmas 3.6 and 3.8. We omit the details.

Suppose $k>1$ and assume that the lemma holds for all members of $\mathcal{I}_{k-1}\left(\left(Q^{*}\right)^{\circ}\right)$. Let $S \in \mathcal{I}_{k}\left(\left(Q^{*}\right)^{\circ}\right)$. There are $f \in \mathcal{F}_{2}$ and $S_{1} \in \mathcal{I}_{k-1}\left(\left(Q^{*}\right)^{\circ}\right)$ such that $S=f\left(S_{1}\right)$. Since $S_{1}$ is connected, so is $f\left(S_{1}\right)$.

Let $p \in T^{\circ} \backslash S=T^{\circ} \backslash f\left(S_{1}\right)$. Since $p \notin f\left(S_{1}\right), f^{-1}(p) \notin S_{1}$. By the induction hypothesis, there exists $(H, K) \in \bigcup_{l=k-1}^{\infty} \mathcal{R}_{l}$ such that $f^{-1}(p) \in H$ and $S_{1} \subseteq T \backslash H$. Note that $(f(H), f(K)) \in \bigcup_{l=k}^{\infty} \mathcal{R}_{l} . f(K)$ is finite and by Lemma 3.6 it separates $f(H)$ from $T \backslash$ $f(H)$. Since $p \in f(H)$ and $f\left(S_{1}\right) \subseteq T \backslash f(H), p$ is not in the same component of $T^{\circ}$ as $f\left(S_{1}\right)$. Thus, $f\left(S_{1}\right)$ is a component of $T^{\circ}$.

Lemma 3.10. $f_{I}\left(\left(Q^{*}\right)^{\circ}\right) \cap f_{J}\left(\left(Q^{*}\right)^{\circ}\right)=\emptyset$ for distinct $I, J \in \mathcal{I}$ and $Q^{\circ} \cap f_{J}\left(\left(Q^{*}\right)^{\circ}\right)=\emptyset$ for all $J \in \mathcal{I}$. In particular, the cardinality of $\mathcal{I}_{k}\left(\left(Q^{*}\right)^{\circ}\right)$ is $(2 m-2)^{k}$.

Proof. By Lemma 3.9 it is enough to show that all elements of

$$
\left\{Q^{\circ}\right\} \cup\left\{f_{J}\left(\left(Q^{*}\right)^{\circ}\right): J \in \mathcal{I}\right\}
$$

are distinct. Note that $\operatorname{diam} Q^{\circ}>\operatorname{diam}\left(Q^{*}\right)^{\circ}$. So, $Q^{\circ}$ and $\left(Q^{*}\right)^{\circ}$ are distinct. Since the maps in $\mathcal{F}$ are contractive, every member of $\mathcal{I}_{1}\left(\left(Q^{*}\right)^{\circ}\right)$ is distinct from both $Q$ and $Q^{*}$. By the open set condition, the elements of $\mathcal{I}_{1}\left(\left(Q^{*}\right)^{\circ}\right)$ are all distinct.

Suppose $k>1$ and assume that the sets in $\mathcal{I}_{k-1}\left(\left(Q^{*}\right)^{\circ}\right)$ are distinct from each other and from members of $\left\{Q^{\circ}\right\} \cup \bigcup_{l=0}^{k-2} \mathcal{I}_{l}\left(\left(Q^{*}\right)^{\circ}\right)$. Since the maps in $\mathcal{F}$ are contractive, every member of $\mathcal{I}_{k}\left(\left(Q^{*}\right)^{\circ}\right)$ is distinct from members of

$$
\left\{Q^{\circ}\right\} \cup \bigcup_{l=0}^{k-1} \mathcal{I}_{l}\left(\left(Q^{*}\right)^{\circ}\right) .
$$

Let $I, J \in \mathcal{I}_{k}$ be distinct. There exist $I^{*}, J^{*} \in \mathcal{I}_{k-1}$ and $-m+1 \leqslant i, j \leqslant m-2$ such that $I=i \oplus I^{*}$ and $J=j \oplus J^{*}$. If $i=j$, then $I^{*}$ and $J^{*}$ are distinct, which implies that $f_{I}\left(\left(Q^{*}\right)^{\circ}\right)=f_{i}\left(f_{I^{*}}\left(\left(Q^{*}\right)^{\circ}\right)\right)$ and $f_{J}\left(\left(Q^{*}\right)^{\circ}\right)=f_{i}\left(f_{J^{*}}\left(\left(Q^{*}\right)^{\circ}\right)\right)$ are distinct. If $i \neq j$, then the open set condition implies that $f_{I}=f_{i}\left(f_{I^{*}}\left(\left(Q^{*}\right)^{\circ}\right)\right)$ and $f_{J}=f_{j}\left(f_{J^{*}}\left(\left(Q^{*}\right)^{\circ}\right)\right)$ are distinct.

Define

$$
U:=Q^{\circ} \bigcup \bigcup_{S \in \mathcal{I}\left(\left(Q^{*}\right)^{\circ}\right)} S
$$

To complete the proof of Proposition 3.5, it suffices to show that $T=\operatorname{cl}(U)$. 
Let $\bar{n}:=\frac{1}{2} n-1$. Define the infinite sum

$$
s:=\sum_{k=0}^{\infty} \frac{p_{k}}{n^{k}},
$$

where the $p_{k}$ satisfy the following Fibonacci-type relations:

$$
p_{0}=1, \quad p_{1}=\bar{n}, \quad p_{k}=\bar{n}\left(p_{k-1}+p_{k-2}\right) \quad \text { for all } k \geqslant 2 .
$$

We leave it for the reader to verify that $s=\mu(Q) / \mu\left(Q_{0}\right)$.

Lemma 3.11. For all $k \geqslant 2, p_{k}=\bar{n}^{k}+\sum_{j=2}^{k} \bar{n}^{j-1} p_{k-j}$.

Proof. We use induction on $k \cdot p_{2}=\bar{n}\left(p_{0}+p_{1}\right)=\bar{n}^{2}+\bar{n}$ and hence the equality holds. Assume that it holds for some $k \geqslant 2$. Then

$$
\begin{aligned}
p_{k+1} & =\bar{n}\left(p_{k}+p_{k-1}\right) \\
& =\bar{n}\left(\bar{n}^{k}+\sum_{j=2}^{k} \bar{n}^{j-1} p_{k-j}\right)+\bar{n} p_{k-1} \\
& =\bar{n}^{k+1}+\sum_{j=2}^{k+1} \bar{n}^{j-1} p_{k+1-j},
\end{aligned}
$$

which completes the induction.

Lemma 3.12.

$$
s=\frac{n^{2}}{n(n-\bar{n})-\bar{n}} .
$$

In particular,

$$
\mu(Q)=\frac{2 n^{5 / 2}}{n^{2}+n+2} .
$$

Proof. Using Lemma 3.11 and reordering the summations, we obtain

$$
\begin{aligned}
s & =1+\frac{\bar{n}}{n}+\sum_{k=2}^{\infty} \frac{1}{n^{k}}\left(\bar{n}^{k}+\sum_{j=2}^{k} \bar{n}^{j-1} p_{k-j}\right) \\
& =\sum_{k=0}^{\infty}\left(\frac{\bar{n}}{n}\right)^{k}+\sum_{k=2}^{\infty} \sum_{j=2}^{k} \frac{\bar{n}^{j-1}}{n^{k}} p_{k-j} \\
& =\frac{n}{n-\bar{n}}+\sum_{\ell=0}^{\infty}\left(\sum_{j=2}^{\infty} \frac{\bar{n}^{j-1}}{n^{j+\ell}}\right) p_{\ell} \\
& =\frac{n}{n-\bar{n}}+\frac{\bar{n}}{n(n-\bar{n})} \sum_{\ell=0}^{\infty} \frac{p_{\ell}}{n^{\ell}} \\
& =\frac{n}{n-\bar{n}}+\frac{\bar{n}}{n(n-\bar{n})} s .
\end{aligned}
$$


Solving the resulting equation for $s$ yields the first required equality. The second equality follows from the facts that $s=\mu(Q) / \mu\left(Q_{0}\right), \mu\left(Q_{0}\right)=\sqrt{n}$ and $\bar{n}=\frac{1}{2} n-1$.

Lemma 3.13. Let $U$ denote the union defined in (3.1). Then $T=\operatorname{cl}(U)$.

Proof. By summing the areas of the essentially disjoint sets in the union as a geometric series, and using the relation $n=2 m$, we obtain

$$
\begin{aligned}
\mu(U) & =\mu(Q)\left[1+\frac{m-1}{n}+\sum_{k=0}^{\infty}(2 m-2)^{k}(2 m-3)(m-1)\left(\frac{1}{n}\right)^{k+2}\right] \\
& =\mu(Q)\left[1+\frac{m-1}{n}+\frac{(2 m-3)(m-1)}{n^{2}} \sum_{k=0}^{\infty}\left(\frac{2 m-2}{n}\right)^{k}\right] \\
& =\mu(Q) \frac{n^{2}+n+2}{4 n} .
\end{aligned}
$$

Now, by Lemma 3.12, $\mu(Q)=\left(2 n^{5 / 2}\right) /\left(n^{2}+n+2\right)$. Combining these results, and using the fact that $\mu(T)=\frac{1}{2} \mu\left(T_{0}\right)=\frac{1}{2} n^{3 / 2}$, we get

$$
\mu(U)=\frac{2 n^{5 / 2}}{n^{2}+n+2} \frac{n^{2}+n+2}{4 n}=\frac{n^{3 / 2}}{2}=\mu(T) .
$$

It follows that $T^{\circ} \subseteq U$ and thus $T=\operatorname{cl}\left(T^{\circ}\right) \subseteq \operatorname{cl}(U)$. On the other hand, it is obvious that $\operatorname{cl}(U) \subseteq T$. Hence $T=\operatorname{cl}(U)$.

Proposition 3.5 now follows by combining Lemmas 3.9 and 3.13 .

To complete the proof of Theorem 1.1, it now remains to show that the closure of each component of $T^{\circ}$ has infinitely many holes.

Lemma 3.14. Let $A$ and $C$ be non-empty subsets of $\mathbb{R}^{2}$ such that $C$ is connected, $\partial C \subseteq A$, and $C \nsubseteq A$. If $B$ is a component of $\mathbb{R}^{2} \backslash A$ and $B \cap C \neq \emptyset$, then $B \subseteq C$.

Proof. Let $B$ be a component of $\mathbb{R}^{2} \backslash A$ such that $B \cap C \neq \emptyset$. By way of contradiction assume that $B \nsubseteq C$. Since $B$ is connected, $B \cap C \neq \emptyset$, and $B \nsubseteq C$, we have $B \cap \partial C \neq \emptyset$. Since $\partial C \subseteq A$, we get $B \cap A \neq \emptyset$, which contradicts the premise that $B \subseteq \mathbb{R}^{2} \backslash A$.

Lemma 3.15. If $S$ is a component of $T^{\circ}$, then $\operatorname{cl}(S)$ has infinitely many holes, i.e. $\mathbb{R}^{2} \backslash$ $\mathrm{cl}(S)$ consists of infinitely many bounded components.

Proof. We first show that $Q$ has infinitely many holes. Given a function $f$, we let $f^{0}$ denote the identity. Recall that $Q_{1}:=\mathcal{H}_{1}\left(Q_{0}\right)$. Define

$$
O:=Q_{0} \cup f_{-m+1}\left(Q_{0}\right) \cup f_{-m+1}\left(Q_{1}\right) \cup f_{-m+1} f_{-1}\left(Q_{1}\right) \cup f_{-m+1} f_{0}\left(Q_{1}\right) \cup \mathcal{H}_{3}\left(Q_{0}\right) .
$$

Note that $O \subseteq Q$ and there is a bounded component $C_{0}$ of $\mathbb{R}^{2} \backslash O$ contained in $T_{0}^{\circ}$. It may also be verified that $\partial C_{0} \subseteq Q$ and $C_{0} \nsubseteq T$. In particular, $C_{0} \nsubseteq Q$. Let $p \in C_{0} \backslash T$. Since $p \notin Q$, there is a component $H_{0}$ of $\mathbb{R}^{2} \backslash Q$ such that $p \in H_{0}$. By Lemma 3.14, $H_{0} \subseteq C_{0} \subseteq T_{0}^{\circ}$. Since $p \in H_{0}, H_{0} \nsubseteq T$. 


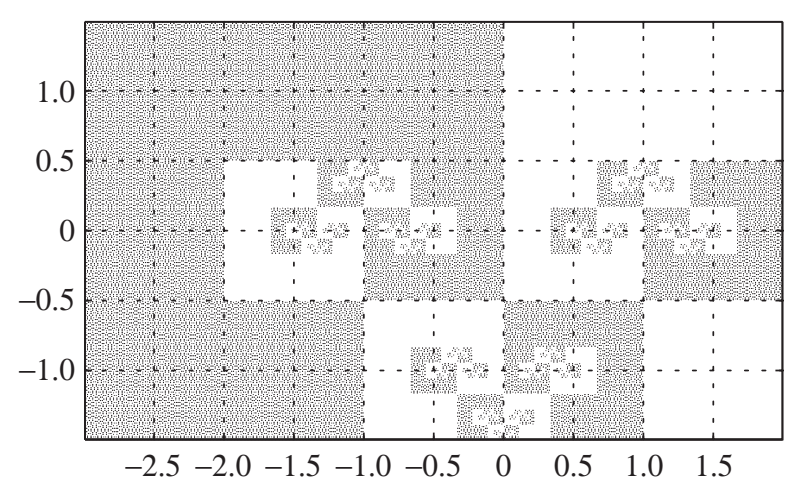

Figure 2. A connected 9-reptile with holes.

Suppose that $k>0$ and assume that $\mathbb{R}^{2} \backslash Q$ has a bounded component $H_{k-1} \subseteq$ $f_{-m+1}^{k-1}\left(T_{0}^{\circ}\right)$ such that $H_{k-1} \not \subset T$. Define $C_{k}:=f_{-m+1}\left(H_{k-1}\right)$. Then $C_{k}$ is connected, $C_{k} \subseteq f_{-m+1}^{k}\left(T_{0}^{\circ}\right)$, and $C_{k} \nsubseteq f_{-m+1}(T)$. By Lemma 2.5,

$$
\operatorname{cl}\left(T \cap\left(f_{-m+1}\left(T_{0}\right)\right)^{\circ}\right)=f_{-m+1}(T) .
$$

So, $C_{k} \nsubseteq \subseteq T$. In particular, $C_{k} \nsubseteq Q$. Since $\partial H_{k-1} \subseteq Q$, the definition of $Q$ implies that $\partial C_{k}=f_{-m+1}\left(\partial H_{k-1}\right) \subseteq f_{-m+1}(Q) \subseteq Q$. Let $q \in C_{k} \backslash T$. Since $q \notin Q$, there is a component $H_{k}$ of $\mathbb{R}^{2} \backslash Q$ such that $q \in H_{k}$. By Lemma 3.14, $H_{k} \subseteq C_{k} \subseteq f_{-m+1}^{k}\left(T_{0}^{\circ}\right)$. Since $q \in H_{k}, H_{k} \nsubseteq \nsubseteq T$.

Since the diameters of the terms of the sequence $\left\{H_{k}\right\}_{k=0}^{\infty}$ go to $0, Q$ has infinitely many holes.

We now show that $Q^{*}$ has infinitely many holes. Let $H$ be a bounded component of $\mathbb{R}^{2} \backslash Q$ such that $H \nsubseteq \subseteq T$. Since $H$ is open, $H \subseteq T_{0}^{\circ}$. Therefore, $f_{0}(H) \subseteq f_{0}\left(T_{0}^{\circ}\right)$ and $f_{0}(H) \nsubseteq f_{0}(T)$. Lemma 2.5 thus yields $f_{0}(H) \nsubseteq Q^{*}$. Moreover, it is obvious that $f_{0}(H)$ is connected and $\partial\left(f_{0}(H)\right) \subseteq Q^{*}$. Let $r \in f_{0}(H) \backslash Q^{*}$. Let $H^{*}$ be the component of $\mathbb{R}^{2} \backslash Q^{*}$ which contains $r$. By Lemma 3.14, $H^{*} \subseteq f_{0}(H)$. Thus, $H^{*}$ is a hole in $Q^{*}$. Since $Q$ has infinitely many holes of decreasing diameter it follows that the same holds for $Q^{*}$.

If $S$ is the closure of a component of $T^{\circ}$ other than $Q$ or $Q^{*}$, then $S$ is similar to $Q^{*}$ by virtue of Lemma 3.9. Thus, $S$ has infinitely many holes.

Lemma 3.15 now completes the proof of Theorem 1.1.

Theorem 1.1 reduces the original question in $[\mathbf{3}, \S \mathrm{C} 17]$ to the following one.

Question 1. Are there 2-reptiles or 3-reptiles in $\mathbb{R}^{2}$ with holes?

\section{Reptiles with other topological properties}

In this section, we use the method in $\S 2$ to construct reptiles with holes and various properties.

For $n$ equal to an odd integer, we can also construct certain $n$-reptiles with holes. The smallest $n$ we can get is 9 , as in the following example. 
Example 4.1. Let $T_{0}=[-3,3] \times\left[-\frac{3}{2}, \frac{3}{2}\right]$ and $\rho=\frac{1}{3}$. Define an IFS $\mathcal{F}=\left\{f_{i}\right\}_{i=1}^{9}$ as

$$
f_{i}(x)= \begin{cases}\rho R\left(\frac{1}{2} \pi\right)(x)+d_{i}, & i=1, \\ \rho R\left(\frac{1}{2} \pi\right) \sigma_{y}(x)+d_{i}, & i=2, \\ \rho x+d_{i}, & i=3,4, \\ \rho \sigma_{y}(x)+d_{i}, & i=5,6 \\ \rho \sigma_{y}(x)+d_{i}, & i=7, \\ \rho R(\pi)(x)+d_{i}, & i=8,9\end{cases}
$$

where

$$
\begin{array}{lll}
d_{1}=d_{2}=\left(-\frac{5}{2}, \frac{1}{2}\right), & d_{3}=d_{5}=(-2,-1), & d_{4}=d_{6}=(-1,1), \\
d_{7}=(0,-1), & d_{8}=(-1,0), & d_{9}=(1,0) .
\end{array}
$$

Then the attractor $T$ (see Figure 2) of $\mathcal{F}$ is a connected 9-reptile whose interior consists of infinitely many components, with the closure of each component consisting of infinitely many holes.

Proof. It is clear that $T_{0}$ satisfies the conditions in (1.4). Let $\mathcal{G}=\left\{\mathrm{id}, \sigma_{y}\right\}$. Then Condition 1.2 holds. One can check directly that

$$
\begin{array}{lll}
f_{1} \sigma_{y}=f_{2}, & f_{2} \sigma_{y}=f_{1}, & f_{3} \sigma_{y}=f_{5}, \\
f_{5} \sigma_{y}=f_{3}, & f_{4} \sigma_{y}=f_{6}, & f_{6} \sigma_{y}=f_{4}, \\
f_{7} \sigma_{y}=\sigma_{y} f_{7}, & f_{8} \sigma_{y}=\sigma_{y} f_{9}, & f_{9} \sigma_{y}=\sigma_{y} f_{8} .
\end{array}
$$

It follows that $\mathcal{F}_{1}:=\left\{f_{i}\right\}_{i=1}^{6}, \mathcal{F}_{2}:=\left\{f_{i}\right\}_{i=7}^{9}$, and Condition 1.3 holds. Thus by Theorem 1.5 (a), $T$ is a 9 -reptile. It is easy to see that, for all $f \in \mathcal{F}, V_{0} \cap f\left(T_{0}\right) \neq \emptyset$, where $V_{0}$ is defined as in (1.8). Thus, by Theorem 1.5(b), $T$ is connected. The rest of the assertions can be proved as in $\S 3$.

To construct $n$-reptiles in $\mathbb{R}^{2}$ whose interior consist of finitely many components, and with the closure of some component having a hole, the smallest $n$ we can obtain is 16 . Note that reflections are not involved in the construction of the following reptile.

Example 4.2. Let $T_{0}=[-8,8] \times[-4,4]$ and $\rho=\frac{1}{4}$. Define an IFS $\mathcal{F}:=\left\{f_{i}\right\}_{i=1}^{16}$ as

$$
f_{i}(x)= \begin{cases}\rho R\left(\frac{1}{2} \pi\right)(x)+d_{i}, & 1 \leqslant i \leqslant 5 \\ \rho R\left(-\frac{1}{2} \pi\right)(x)+d_{i}, & 6 \leqslant i \leqslant 10 \\ \rho x+d_{i}, & 11 \leqslant i \leqslant 13 \\ \rho R(-\pi) x+d_{i}, & 14 \leqslant i \leqslant 16\end{cases}
$$

where

$$
\begin{aligned}
& d_{1}=d_{6}=(-7,-2), \quad d_{2}=d_{7}=(-7,2), \quad d_{3}=d_{8}=(-5,0), \quad d_{4}=d_{9}=(-1,0), \\
& d_{5}=d_{10}=(3,0), \quad d_{11}=d_{14}=(-4,-3), \quad d_{12}=d_{15}=(-4,3), \quad d_{13}=d_{16}=(0,3) \text {. }
\end{aligned}
$$




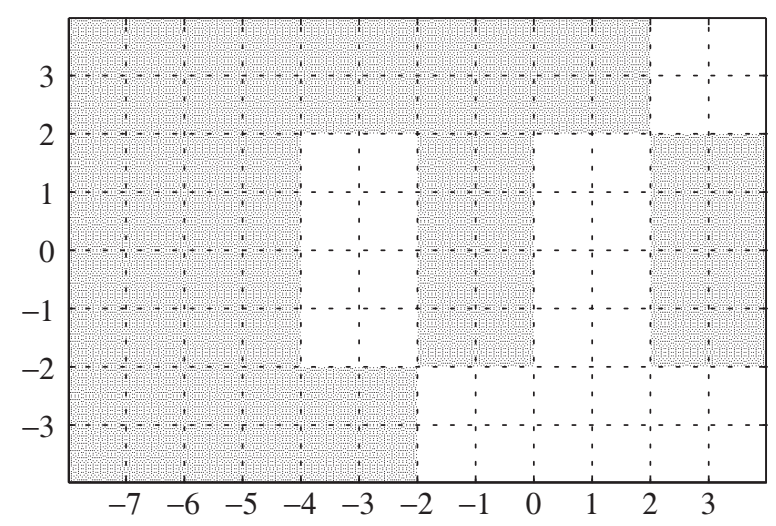

Figure 3. A connected 16-reptile whose interior consists of two components, with the closure of one of them being a disc and that of the other having a hole.

Then the attractor $T$ of $\mathcal{F}$ is a connected 16-reptile whose interior consists of two components, with the closure of exactly one of them having a hole (see Figure 3).

Proof. Let $\mathcal{G}=\{\operatorname{id}, R(-\pi)\}$. Then Condition 1.2 clearly holds. For $1 \leqslant i \leqslant 5$, $f_{i} R(-\pi)=f_{i+5}$, which implies that $f_{i+5} R(-\pi)=f_{i}$. For $11 \leqslant i \leqslant 13$, we have $f_{i} R(-\pi)=f_{i+3}$, which implies that $f_{i+3} R(-\pi)=f_{i}$. Hence $\mathcal{F}_{1}=\mathcal{F}$ and Condition 1.3 also holds. Thus $T$ is a 16 -reptile.

For all $f \in \mathcal{F}, V_{0} \cap f\left(T_{0}\right) \neq \emptyset$. Hence $T$ is connected. The last assertion is obvious.

In view of Example 4.2, it is natural to ask the following questions.

Question 2. What is the smallest $n$ such that a connected $n$-reptile in $\mathbb{R}^{2}$, whose interior consists of finitely many components, has a hole? What is the smallest $n$ such that a connected piecewise polygonal $n$-reptile in $\mathbb{R}^{2}$ has a hole?

We now construct a reptile whose interior consists of infinitely many components, with the closure of some components having holes and the closure of the others being topological discs.

Example 4.3. Let $T_{0}=[-4,4] \times[-2,2]$ and $\rho=\frac{1}{4}$. Define and IFS $\mathcal{F}=\left\{f_{i}\right\}_{i=1}^{16}$ as

$$
f_{i}(x)= \begin{cases}\rho R\left(\frac{1}{2} \pi\right)(x)+d_{i}, & 1 \leqslant i \leqslant 5 \\ \rho R\left(\frac{1}{2} \pi\right) \sigma_{y}(x)+d_{i}, & 6 \leqslant i \leqslant 10 \\ \rho x+d_{i}, & i=11,12 \\ \rho \sigma_{y}(x)+d_{i}, & i=13,14 \\ \rho R\left(\frac{1}{2} \pi\right)(x)+d_{i}, & i=15, \\ \rho R\left(-\frac{1}{2} \pi\right)(x)+d_{i}, & i=16\end{cases}
$$




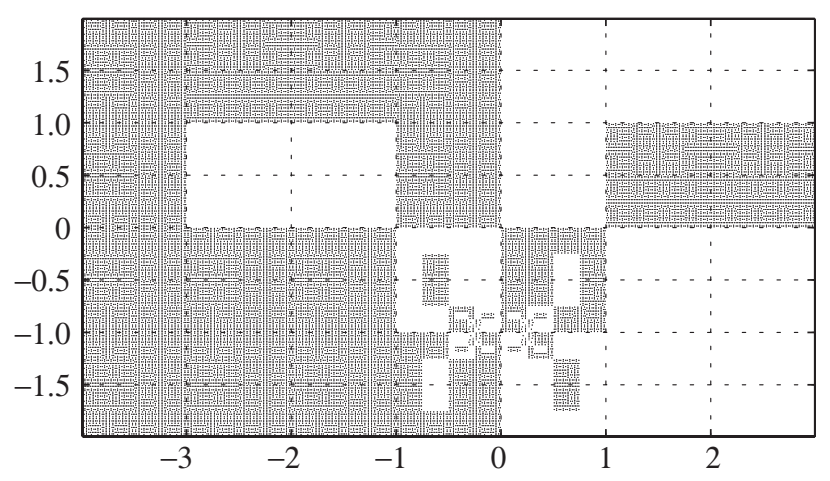

Figure 4. The reptile in Example 4.3.

where

$$
\begin{aligned}
d_{1} & =d_{6}=\left(-\frac{3}{2},-1\right), & d_{2} & =d_{7}=\left(-\frac{5}{2},-1\right), & d_{3} & =d_{8}=\left(-\frac{7}{2},-1\right), \\
d_{4} & =d_{9}=\left(-\frac{7}{2}, 1\right), & d_{5} & =d_{10}=\left(-\frac{1}{2}, 1\right), & d_{11} & =d_{13}=\left(-2, \frac{3}{2}\right), \\
d_{12} & =d_{14}=\left(2, \frac{1}{2}\right), & d_{15} & =\left(-\frac{1}{2},-1\right), & d_{16} & =\left(\frac{1}{2},-1\right) .
\end{aligned}
$$

Then the attractor $T$ (see Figure 4 ) of $\mathcal{F}$ is a connected 16-reptile whose interior consists of infinitely many components, with the closure of some of them having holes and the closure of the others being topological discs.

Proof. Let $\mathcal{G}=\left\{\mathrm{id}, \sigma_{y}\right\}$; thus Condition 1.2 holds. We verify directly that, for $1 \leqslant$ $i \leqslant 5, f_{i} \sigma_{y}=f_{i+5} \in \mathcal{F}$, which implies that $f_{i+5} \sigma_{y}=f_{i}$. For $i=11$ or 12 , we have $f_{i} \sigma_{y}=$ $f_{i+2} \in \mathcal{F}$, which implies that $f_{i+2} \sigma_{y}=f_{i}$. Also, it is easy to check that $f_{15} \sigma_{y}=\sigma_{y} f_{16}$, which implies $f_{16} \sigma_{y}=\sigma_{y} f_{15}$. Thus, $\mathcal{F}_{1}=\left\{f_{i}\right\}_{i=1}^{14}, \mathcal{F}_{2}=\left\{f_{i}\right\}_{i=15}^{16}$, and Condition 1.3 is satisfied. Therefore, $T$ is a 16-reptile.

For all $f \in \mathcal{F}, V_{1} \cap f\left(T_{0}\right) \neq \emptyset$. Hence $T$ is connected. We omit the proofs of the rest of the assertions.

It is not true that if the interior of a reptile consists of infinitely many components, with the closure of some having holes, then the closure of these components must contain infinitely many holes. The following is a counterexample.

Example 4.4. Let $T_{0}=[-6,6] \times[-3,3]$ and $\rho=\frac{1}{6}$. Define and IFS $\mathcal{F}=\left\{f_{i}\right\}_{i=1}^{36}$ as

$$
f_{i}(x)= \begin{cases}\rho \sigma_{y}(x)+d_{i}, & 1 \leqslant i \leqslant 7 \\ \rho R\left(\frac{1}{2} \pi\right) x+d_{i}, & 8 \leqslant i \leqslant 16 \\ \rho R\left(\frac{1}{2} \pi\right) \sigma_{y}(x)+d_{i}, & 17 \leqslant i \leqslant 25 \\ \rho x+d_{i}, & 26 \leqslant i \leqslant 34 \\ \rho \sigma_{y}(x)+d_{i}, & i=35 \\ \rho R(\pi)(x)+d_{i}, & i=36\end{cases}
$$




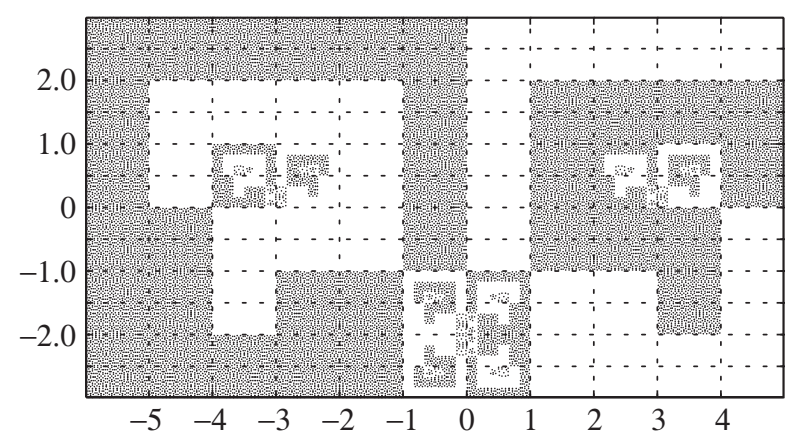

Figure 5. A connected 36-reptile whose interior consists of infinitely many components and the closure of each component contains finitely many holes.

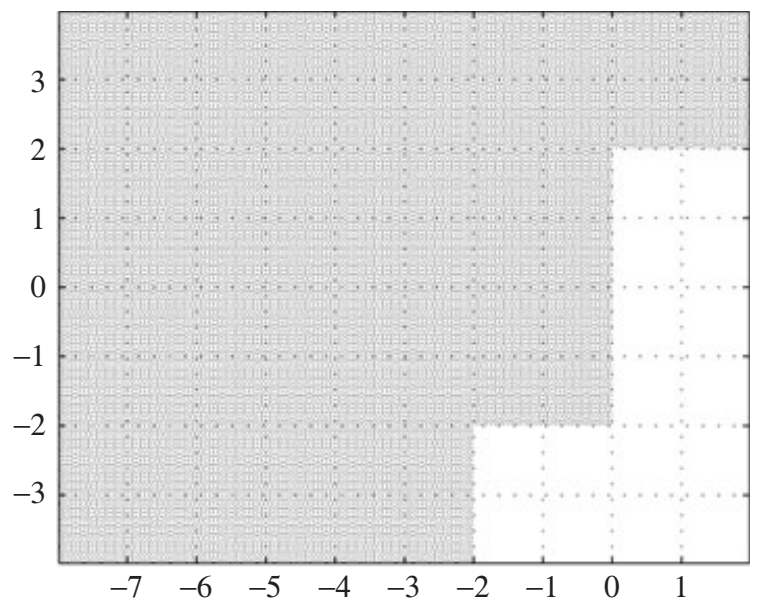

Figure 6. The 16-reptile $S$ used in the construction of the reptile $R$ in $\mathbb{R}^{3}$.

where

$$
\begin{aligned}
d_{1} & =d_{26}=\left(-4,-\frac{5}{2}\right), & d_{2} & =d_{27}=\left(-4, \frac{5}{2}\right), & d_{3} & =d_{28}=\left(-2,-\frac{5}{2}\right), \\
d_{4} & =d_{29}=\left(-2,-\frac{3}{2}\right), & d_{5} & =d_{30}=\left(-2, \frac{5}{2}\right), & d_{6} & =d_{31}=\left(2,-\frac{1}{2}\right), \\
d_{7} & =d_{32}=\left(3, \frac{3}{2}\right), & d_{8} & =d_{17}=\left(-\frac{11}{2},-2\right), & d_{9} & =d_{18}=\left(-\frac{11}{2}, 0\right), \\
d_{10} & =d_{19}=\left(-\frac{11}{2}, 2\right), & d_{11} & =d_{20}=\left(-\frac{9}{2},-1\right), & d_{12} & =d_{21}=\left(-\frac{1}{2}, 0\right), \\
d_{13} & =d_{22}=\left(-\frac{1}{2}, 2\right), & d_{14} & =d_{23}=\left(\frac{3}{2}, 1\right), & d_{15} & =d_{24}=\left(\frac{7}{2},-1\right), \\
d_{16} & =d_{25}=\left(\frac{9}{2}, 1\right), & d_{33} & =\left(-3, \frac{1}{2}\right), & d_{34} & =\left(3, \frac{1}{2}\right), \\
d_{35} & =\left(0,-\frac{3}{2}\right), & d_{36} & =\left(0,-\frac{5}{2}\right) . & &
\end{aligned}
$$

Then the attractor $T$ (see Figure 5 ) of $\mathcal{F}$ is a connected 36-reptile whose interior consists of infinitely many components, with the closure of each component consisting of finitely many holes. 
Proof. It is clear that $\mathcal{G}=\left\{\mathrm{id}, \sigma_{y}\right\}$ satisfies Condition 1.2. We first note that, for $1 \leqslant i \leqslant 7, f_{i} \sigma_{y}=f_{i+25}$, which implies that $f_{i+25} \sigma_{y}=f_{i}$. Next, we can check directly that, for $8 \leqslant i \leqslant 16, f_{i} \sigma_{y}=f_{i+9}$, which implies that $f_{i+9}=f_{i} \sigma_{y}$. Lastly, we verify directly that $f_{35} \sigma_{y}=\sigma_{y} f_{35}$ and $f_{36} \sigma_{y}=\sigma_{y} f_{36}$; moreover, $f_{33} \sigma_{y}=\sigma_{y} f_{34}$, which implies that $f_{34} \sigma_{y}=\sigma_{y} f_{33}$. Thus $\mathcal{F}_{1}=\left\{f_{i}\right\}_{i=1}^{32}, \mathcal{F}_{2}=\mathcal{F} \backslash \mathcal{F}_{1}$, and Condition 1.3 holds. Therefore, $T$ is a 36 -reptile.

For all $f \in \mathcal{F}, V_{1} \cap f\left(T_{0}\right) \neq \emptyset$. Hence $T$ is connected. We omit the proofs of the rest of the assertions.

By modifying and extending the reptile $T$ in Example 4.2 (see Figure 3), we can obtain a reptile in $\mathbb{R}^{3}$ with a hole. Let $S$ be the 16-reptile shown in Figure 6. Define

$$
R:=(S \times[-2,-1]) \cup(T \times[-1,0]) \cup(T \times[0,1]) \cup(S \times[1,2]) .
$$

Then $R$ is a 64 -reptile in $\mathbb{R}^{3}$ whose interior consists of two components, with the closure of one them having a hole.

Acknowledgements. The authors thank the referee for some valuable suggestions.

\section{References}

1. C. BAndt, Self-similar sets, 5, Integer matrices and fractal tilings of $\mathbb{R}^{n}$, Proc. Am. Math. Soc. 112 (1991), 549-562.

2. C. BAndt And Y. WAng, Disk-like self-affine tiles in $\mathbb{R}^{2}$, Discrete Comput. Geom. 26 (2001), 591-601.

3. H. T. Croft, K. J. FAlconer And R. K. GuY, Unsolved problems in geometry (Springer, 1991).

4. K. J. FALCONER, Fractal geometry. Mathematical foundations and applications (Wiley, 1990).

5. B. Grünbaum And G. C. Shephard, Tilings and patterns (W. H. Freeman, New York, 1987).

6. M. HAtA, On the structure of self-similar sets, Jpn. J. Appl. Math. 2 (1985), 381-414.

7. J. E. Hutchinson, Fractals and self-similarity, Indiana Univ. Math. J. 30 (1981), 713747.

8. J. C. Lagarias And Y. WANG, Self-affine tiles in $\mathbb{R}^{n}$, Adv. Math. 121 (1996), 21-49.

9. J. LuO, H. RAO AND B. TAN, Topological structure of self-similar sets, Fractals 10 (2002), 223-227.

10. S.-M. NGai AND T.-M. TANG, Topology of connected self-similar tiles in the plane with disconnected interiors, Topol. Applic. 150 (2005), 139-155.

11. A. Schief, Separation properties for self-similar sets, Proc. Am. Math. Soc. 122 (1994), 111-115. 